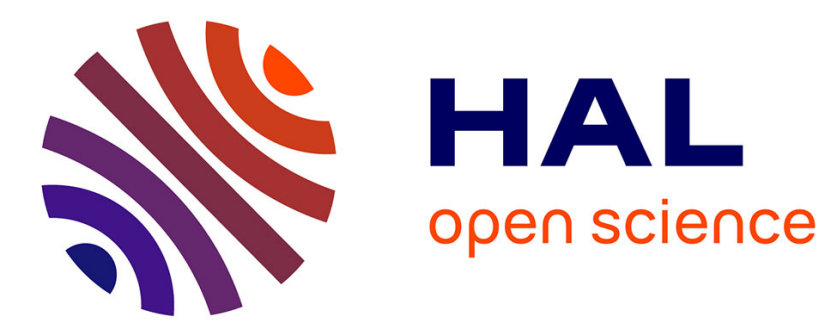

\title{
Cauchy's Flux Theorem in Light of Geometric Integration Theory
}

Guy Rodnay, Reuven Segev

\section{To cite this version:}

Guy Rodnay, Reuven Segev. Cauchy's Flux Theorem in Light of Geometric Integration Theory. Journal of Elasticity, 2003, 71 (1-3), pp.183-203. hal-00956979

\section{HAL Id: hal-00956979 \\ https://hal.science/hal-00956979}

Submitted on 7 Mar 2014

HAL is a multi-disciplinary open access archive for the deposit and dissemination of scientific research documents, whether they are published or not. The documents may come from teaching and research institutions in France or abroad, or from public or private research centers.
L'archive ouverte pluridisciplinaire HAL, est destinée au dépôt et à la diffusion de documents scientifiques de niveau recherche, publiés ou non, émanant des établissements d'enseignement et de recherche français ou étrangers, des laboratoires publics ou privés. 


\title{
CAUCHY'S FLUX THEOREM IN LIGHT OF GEOMETRIC INTEGRATION THEORY
}

\author{
G. RODNAY AND R. SEGEV
}

\begin{abstract}
This work presents a formulation of Cauchy's flux theory of continuum mechanics in the framework of geometric integration theory as formulated by $\mathrm{H}$. Whitney and extended recently by J. Harrison. Starting with convex polygons, one constructs a formal vector space of polyhedral chains. A Banach space of chains is obtained by a completion process of this vector space with respect to a norm. Then, integration operators, cochains, are defined as elements of the dual space to the space of chains. Thus, the approach links the analytical properties of cochains with the corresponding properties of the domains in an optimal way. The basic representation theorem shows that cochains may be represented by forms. The form representing a cochain is a geometric analog of a flux field in continuum mechanics.
\end{abstract}

\section{INTRODUCTION}

The Cauchy Theorem for the existence of stresses and fluxes is one of the fundamental results of continuum mechanics. Over the years, research work contributed to the subject by making the proof more rigorous, by weakening the postulates needed to prove the theorem, by extending the circumstances under which it is valid, and by proving the existence of stresses and fluxes using alternative methods and approaches.

In terms of scalar fluxes in space, the basic notions of flux theory may be described as follows. One considers the total flux $T(\partial B)$ of an extensive property $P$ through the boundary $\partial B$ of the region $B$ in a three dimensional Euclidean space. The total flux is assumed to be given as an integral of the flux density $t_{B}$ associated with the region $B$, a scalar field defined on $\partial B$, in the form

$$
T(\partial B)=\int_{\partial B} t_{B} d A .
$$

The dependence of the flux density $t_{B}$ on the region $B$ is considered next and it is assumed that at each point $p, t_{B}(p)$ depends on $B$ only through the unit normal vector $n$ to $\partial B$ at $p$ so one writes $t(p, \boldsymbol{n})$ for the corresponding value. Then, one assumes that the total flux is balanced by the rate of decrease of the total amount of the property $P$ in $B$ as given in terms of an integral of a scalar field $b$ over $B$ so

$$
\int_{\partial B} t_{B} d A=-\int_{B} b d V .
$$

Assuming that the dependence of $t(p, \boldsymbol{n})$ on $p$ is continuous one proves Cauchy's theorem asserting that $t(p, \boldsymbol{n})$ depends linearly on $\boldsymbol{n}$. Thus, there is a vector field $\boldsymbol{\tau}$ such that $t=\boldsymbol{\tau} \cdot \boldsymbol{n}$, where the dependence on $p$ was suppressed in the notation.

Considering smooth regions such that Gauss' theorem may be applied, the balance may be written in the form of a differential equation as $\operatorname{div} \tau+b=0$.

Key words and phrases. Continuum mechanics, flux, Cauchy's theorem, geometric integration, chains, cochains, flat, sharp, natural. 1991/95 Math. Subject Class. 73A05, 58A05. 
The first major contribution within the continuum mechanics community was made by Noll in 1957 [11]. Noll was able to prove the dependence of the flux on the normal vector, using a weaker assumption of locality, namely, the flux density $t_{B}(p)$ is equal for two regions if the intersection of their boundaries contains an open neighborhood of $p$.

Gurtin and Williams in [5] and later works [12, 6, 13] use alternative assumptions, bi-additivity and boundedness, to obtain both locality and the representation of total flux in terms of the integral of a flux density. Specifically, assuming that the collection of admissible regions has the structure of a Boolean lattice, it is required that $T$ be given in terms of a mapping of pairs of bodies $I(A, B)$, so $T(A)=I(A, B)$ if $B$ is the complement of $A$. For separate (disjoint) domains $A$ and $B, I$ satisfies $I(A \curlyvee B, C)=I(A, C)+$ $I(B, C)$ and $I(C, A \curlyvee B)=I(C, A)+I(C, B)$. Then, it is assumed that $|I(A, B)| \leq$ $l$ area $(\partial A \cap \partial B)+k$ volume $(A)$ - the boundedness assumption - to obtain locality. To prove Cauchy's theorem it is assumed also that $I(A, B)=-I(B, A)$ and that the dependence of $\tau(p, \boldsymbol{n})$ on $p$ is continuous.

In [4] Gurtin and Martins prove the linearity in $\boldsymbol{n}$ of $t(p, \boldsymbol{n})$ almost everywhere, while using similar additivity and boundedness assumptions but relaxing the hypothesis that $t(p, \boldsymbol{n})$ is a continuous function of $p$.

In $[20,21]$ Šilhavý uses a weak approach to prove the existence of stress tensors or flux vectors. Admissible bodies are sets of finite perimeter in $E^{n}$, and the assumptions and results pertain to "almost every subbody" in a way which allows singularities. The resulting flux vector $\boldsymbol{\tau}$ has an $L^{p}$ weak divergence.

Degiovanni et al. [1] generalize [20,21] by considering flux mappings $T$ whose corresponding flux vector fields $\tau$ are only locally integrable. The field $b=-\operatorname{div} \tau$ is meaningful only in the weak sense.

In order to present results that hold for domains and flux fields that are increasingly irregular, the works cited above rely on geometric measure theory of Federer [2] and de Giorgi (see [14]). For example, tools of geometric measure theory are used for choosing a universe of bodies, for a measure theoretic definition of the normal vector, and for using generalizations of Gauss' theorem to such irregular domains.

Another approach for proving Cauchy's theorem directly from an integral balance equation is introduced in [3]. In this paper a variational approach it taken to prove the linear dependence on the normal starting from a weaker locality postulate.

Stress theory for manifolds that are not equipped with a metric is presented in [15] from a weak point of view. Forces are defined as elements of the dual space of the Banach space of $C^{k}$-sections of a vector bundle over the body. Stresses are Borel measures valued in the dual of a jet bundle and they represent forces using a representation theorem. Further analytical aspects of the theory are presented in [18]. In particular, as the theory introduces continuum mechanics of order $k$ as corresponding to the space of $C^{k}$-sections, general consistency conditions that are analogous to Cauchy's postulates are formulated for arbitrary values of $k$ and for stresses as irregular as Borel measures. In [16], and the following [19] and [17] the analog of the classical Cauchy theorem is presented for differentiable manifolds.

In 1947 and 1948 Whitney [22] and Wolfe [24] presented a geometric theory of $r$ dimensional integration in an $n$-dimensional Euclidean space. A comprehensive treatment [23] of the theory was published by H. Whitney in 1957. While geometric measure theory received a lot of attention because of its relevance to the Plateau problem, the mathematical work continuing Whitney's geometric integration theory is limited. In [7] and the following $[8,9,10] \mathrm{J}$. Harrison made important extensions to Whitney's work. To the best 
of our knowledge, Whitney's abstract geometric integration theory was never used in the formulation of Cauchy flux theory in continuum mechanics.

It is our objective here to present the Cauchy flux theory from the point of view of geometric integration. In addition to offering a different approach to flux theory, the following features make it eminently suitable. Firstly, the theory considers various aspects namely, the collection of domains, integration, Stokes' theorem (the analog of Gauss' theorem), and fluxes, from a unified point of view. The properties and degrees of regularity of the various variables are linked. Thus, one may consider less regular domains if one is willing to consider smoother fluxes. In fact, the regions may be as irregular as the Dirac measure and its derivatives if one is willing to admit differentiable flux fields. On the other hand, the flux fields may be as irregular as essentially bounded and measurable functions when the boundaries are as irregular as the graph of an $L^{1}$-mapping. The way the theory is constructed, the relation between the regularity properties of domains and fluxes is optimal in the following sense. The class of domains is the largest class for which the evaluation of the various fluxes is continuous. Conversely, the class of fluxes is the largest class such that the total fluxes depend continuously on the domains.

The codimension, $n-r$, is not limited to the value of 1 as in regular Cauchy flux theory. It follows that the theory may be used to formulate flux theory on membranes, strings etc. Furthermore, the theory does not require that the $r$-dimensional domains be smooth. In fact, it permits for example the calculation of flux through a 1-dimensional "arc" on a 2dimensional domain in $\mathbb{R}^{3}$ which is itself the graph of an $L^{1}$-mapping. In other words, not only the boundary is irregular, but so is the domain itself. Finally, the construction of continuous chains creates a bridge between the classical and weak formulation of the theory.

The elegance of the structure enables its description in just a few sentences. One starts with the building blocks, $r$-dimensional oriented cells (convex polygons) in an $n$ dimensional Euclidean space $E^{n}$. Then, the formal vector space of linear combinations of $r$-cells is considered, where two linear combinations $A$ and $B$ are identified if they may be further subdivided to obtain a common subdivision. The elements of this vector space are called polyhedral $r$-chains. Then, the space of polyhedral chains is completed with respect to a norm to obtain a Banach space. The elements of the resulting complete space are called either flat, sharp, or natural $r$-chains depending on the norms used, and chains collectively. Integration operators are referred to as $r$-cochains and they are defined as continuous linear operators on the space of chains.

The application of geometric integration theory to Cauchy flux theory is based on the identification of a total flux operator on regions with a cochain. In other words, a cochain is analogous to a total flux operator acting on the various domains to produce real numbers. In the case of traditional continuum mechanics, the total flux is regarded a 2-cochain in $E^{3}$. The analog of Cauchy's flux theorem is a representation theorem stating that a cochain may be represented by an $r$-form, an antisymmetric $r$-tensor in $E^{n}$, using integration. As mentioned earlier, the analytical properties of chains and forms representing cochains are determined by the norm used. The topology on the space of chains allows one to extend various operations, e.g., integrals and boundaries, from polyhedral chains to the chains obtained as limits of sequences.

Federer [2, pp. 367-378] introduces flat chains as currents, roughly, continuous linear functionals on the space of smooth forms with compact supports-the geometric analogs of Schwartz distributions, and defines the flat norm as the norm induced on the dual space by the norm $\|\phi\|=\sup _{p}\{|\phi(p)|,|d \phi(p)|\}$ on the space of smooth forms. While Federer's 
treatment of flat chains is concise and elegant, it does not contain the analogs for sharp and natural chains. In addition, it seems to us that Whitney's approach is closer in spirit to the traditional approach of continuum mechanics. Furthermore, as Federer states in [2, p. 378] his main interest has been in chains while Whitney's main concern has been with cochains - the objects representing Cauchy fluxes of continuum mechanics.

It is noted that the expression for the representation of cochains in terms of forms also applies on general manifolds rather than a Euclidean space. In addition, while the definitions of the various norms utilize the metric structure of $E^{n}$, the various topological spaces of chains remain invariant under diffeomorphisms. This suggests an extension of the theories to general manifolds. However, a formal presentation of such a theory is not available yet and will not be considered here.

Thus, the basic constructions, results, and applications to Cauchy flux theory are described below. For details of the mathematical constructions and proofs see [23] and $[8,9]$. We start in Section 2 with the basic building blocks: polyhedral chains and integration on polyhedral chains. Section 3 considers the construction of the various Banach spaces of chains and Section 4 presents the definitions and basic properties associated with cochains - the analogs of the Cauchy flux operators. The Cauchy theorem of fluxes is implied by the representation theorem of cochains by forms as presented in Section 5. Finally, Section 6 considers the extension of the exterior derivative to non-smooth forms through the notion of a coboundary, and the resulting local balance equation.

\section{CHAINS AND INTEGRATION}

2.1. Cells and polyhedral chains. We start with a review of the basic definitions related to integration on chains in an $n$-dimensional Euclidean space $E^{n}$ whose associated vector space is $V$. A cell, $\sigma$, is a non empty bounded subset of $E^{n}$ expressed as an intersection of a finite collection of half spaces. The plane of $\sigma$ is the smallest affine subspace containing $\sigma$, and the dimension of $\sigma$ is the dimension of its plane. We refer to $r$-dimensional cells as $r$-cells.

An oriented $r$-cell is an $r$-cell with a choice of one of the two orientations of the vector space associated with its plane. The cell $-\sigma$ is the cell that contains the same points as $\sigma$ but has the opposite orientation. The boundary of an oriented $r$-cell, $\partial \sigma$, is a collection of oriented $(r-1)$-cells. The boundary of a 1-cell consists of two points, and 0-cell has no boundary. The orientations of the cells that make up the boundary $\partial \sigma$ are determined by the orientation of $\sigma$, in the following way. Given a cell $\sigma^{\prime} \subset \partial \sigma$, let $v_{2}, \ldots, v_{r}$ be a collection of $r-1$ independent vectors that belong to the plane of $\sigma^{\prime}$. Then, this collection is positively oriented if given a vector $v_{1}$ at $\sigma^{\prime}$ that belongs to the plane of $\sigma$ and points out of $\sigma$, the collection $\left(v_{1}, \ldots, v_{r}\right)$ is positively oriented relative to $\sigma$. The boundary of a 1-cell oriented by the vector $p q$, consists of the two 0 -cells $q$ positive and $p$ negative.

Oriented cells are the building blocks of chains. A polyhedral $r$-chain in $E^{n}$ is an element of the vector space spanned by formal linear combinations of $r$-cells, together with the following properties. 1) The polyhedral chain $1 \sigma$ is identified with the cell $\sigma .2$ ) We associate multiplication of a cell by -1 with the operation of inversion of orientation, i.e., $-1 \sigma=-\sigma$. 3) If an oriented cell $\sigma$ is cut into several cells, $\sigma_{1}, \ldots, \sigma_{m}$, then $\sigma$ and $\sigma_{1}+\ldots+\sigma_{m}$ are identified as polyhedral chains. Thus, we identify the union of oriented $r$-cells having disjoint interiors with the polyhedral $r$-chain which is the sum of the $r$-cells. Polyhedral 0 -chains are expressions of the form $\sum a_{i} p_{i}$, where $p_{i}$ are points. The boundary of a cell is thus a chain, the sum of the various oriented cells that make up the boundary as above. 
The space of polyhedral $r$-chains in $E^{n}$ is now an infinite-dimensional vector space denoted by $\mathcal{A}_{r}\left(E^{n}\right)$. The boundary of a polyhedral $r$-chain $A=\sum a_{i} \sigma_{i}$, is a polyhedral $(r-1)$-chain defined to be $\partial A=\sum a_{i} \partial \sigma_{i}$. The boundary of a polyhedral 0 -chain is 0 . Note that by this definition $\partial$ is a linear operator $\mathcal{A}_{r}\left(E^{n}\right) \longrightarrow \mathcal{A}_{r-1}\left(E^{n}\right)$.

2.2. Multivectors. A simple $r$-vector in $V$ is defined in a formal way, to be an expression of the form $v_{1} \wedge \cdots \wedge v_{r}$, where $v_{i} \in V$, the vector space associated with $E^{n}$. We set $r$ vectors in $V$ to be elements of the vector space $V_{r}$ of formal linear combinations of simple $r$-vectors, together with the following properties:

$$
\begin{aligned}
& \text { (1) } v_{1} \wedge \cdots \wedge\left(v_{i}+v_{i}^{\prime}\right) \wedge \cdots \wedge v_{r} \\
& =v_{1} \wedge \cdots \wedge v_{i} \wedge \cdots \wedge v_{r}+v_{1} \wedge \cdots \wedge v_{i}^{\prime} \wedge \cdots \wedge v_{r} \\
& \text { (2) } v_{1} \wedge \cdots \wedge\left(a v_{i}\right) \wedge \cdots \wedge v_{r}=a\left(v_{1} \wedge \cdots \wedge v_{i} \wedge \cdots \wedge v_{r}\right) \text {; } \\
& \text { (3) } v_{1} \wedge \cdots \wedge v_{i} \wedge \cdots \wedge v_{j} \wedge \cdots \wedge v_{r} \\
& =-v_{1} \wedge \cdots \wedge v_{j} \wedge \cdots \wedge v_{i} \wedge \cdots \wedge v_{r}
\end{aligned}
$$

1 -vectors are just vectors, and 0 -vectors are defined to be real numbers. It is noted that any $r$-vector can be written in various equivalent ways. The various identifications above, in particular the antisymmetry, imply that dimension of the space of $r$-vectors is

$$
\operatorname{dim} V_{r}=\frac{n !}{(n-r) ! r !},
$$

where $n$ is the dimension of $V$. If $r>n$ then $V_{r}$ is empty. Given a basis $\left\{e_{i}\right\}$ of $V$, the $r$-vectors $\left\{e_{\lambda_{1} \ldots \lambda_{r}}=e_{\lambda_{1}} \wedge \cdots \wedge e_{\lambda_{r}}\right\}$, such that $1 \leq \lambda_{1}<\cdots<\lambda_{r} \leq n$, form a basis of $V_{r}$.

Given an oriented $r$-simplex $\sigma$ in $E^{n}$, with vertices $p_{0} \ldots p_{r}$, the $r$-vector of $\sigma$, denoted by $\{\sigma\}$, is defined to be $\{\sigma\}=v_{1} \wedge \cdots \wedge v_{r} / r$ !, where the vectors $v_{i}$ are defined by $v_{i}=p_{i}-p_{0}$ and are ordered in such a way that they belong to the orientation of $\sigma$. It is noted that in case $\left\{\sigma_{1}\right\}=a\left\{\sigma_{2}\right\}$ for two $r$-simplexes $\sigma_{1}$ and $\sigma_{2}$, then, the ratio between the $r$-dimensional volumes of the two simplexes relative to any metric is $|a|$. The $r$-vector of $a$ polyhedral $r$-chain $A=\sum a_{i} \sigma_{i}$, where $\sum a_{i} \sigma_{i}$ is a simplicial subdivision of $A$, is defined by $\left\{\sum a_{i} \sigma_{i}\right\}=\sum a_{i}\left\{\sigma_{i}\right\}$. Clearly, this defines the $r$-vectors of $r$-cells too, as $r$-cells are particular polyhedral $r$-chains.

2.3. Multi-covectors. The dual space of $V_{r}$ is denoted by $V^{r}$ and its elements are referred to as $r$-covectors. We now show how $r$-covectors can be expressed using covectors. We denote by $V^{*}$ the dual space of $V$, and by $V_{r}^{*}$ the space which is constructed exactly like the space $V_{r}$, but using the (co-) vectors of $V^{*}$. Hence, elements of $V_{r}^{*}$ are expressions of the form $\sum a_{i} f^{i 1} \wedge \cdots \wedge f^{i r}$, where $f^{i j} \in V^{*}$. The scalar product of elements of $V_{r}^{*}$ and elements of $V_{r}$ is defined by

$$
\begin{aligned}
\left(f^{1} \wedge \cdots \wedge f^{r}\right) \cdot\left(v_{1} \wedge \cdots \wedge v_{r}\right) & =\sum_{\lambda} \epsilon^{\lambda_{1} \ldots \lambda_{r}} f^{1}\left(v_{\lambda_{1}}\right) \cdots f^{r}\left(v_{\lambda_{r}}\right) \\
& =\operatorname{det}\left(\begin{array}{ccc}
f^{1}\left(v_{1}\right) & \cdots & f^{1}\left(v_{r}\right) \\
\cdots & \cdots & \cdots \\
f^{r}\left(v_{1}\right) & \cdots & f^{r}\left(v_{r}\right)
\end{array}\right),
\end{aligned}
$$

for simple vectors, and extends linearly to the vector spaces. Here, $\lambda=\left\{\lambda_{1}, \ldots, \lambda_{r}\right\}$ ranges over the set of all permutations of $(1, \ldots, r)$, and $\epsilon^{\lambda_{1} \ldots \lambda_{r}}$ is the alternating symbol. Any element $\bar{\tau}$ of $V_{r}^{*}$ may be identified with an element $\tau$ of $V^{r}$ by $\tau(\alpha)=\bar{\tau} \cdot \alpha$ for 
any $r$-multivector $\alpha$. Furthermore, an element $\tau$ of $V^{r}$ may be regarded as an alternating multilinear form $\tilde{\tau}$ by

$$
\tau\left(v_{1} \wedge \cdots \wedge v_{r}\right)=\tilde{\tau}\left(v_{1}, \ldots, v_{r}\right)
$$

2.4. Integration of forms over polyhedral chains. The natural integrands over $r$-chains are $r$-forms. An $r$-form in a set $Q \subset E^{n}$ is an $r$-covector valued mapping defined in $Q$. An $r$-form is continuous if its components are continuous functions. The Riemann integral of a continuous $r$-form $\tau$ over an $r$-simplex $\sigma$ is defined as

$$
\int_{\sigma} \tau=\lim _{k \rightarrow \infty} \sum_{\sigma_{k i} \in \mathcal{S}_{k} \sigma} \tau\left(p_{k i}\right) \cdot\left\{\sigma_{k i}\right\},
$$

where $\mathcal{S}_{k} \sigma$ is a sequence of simplicial subdivisions $\sigma_{k i}$ of $\sigma$ with mesh $\rightarrow 0$, and each $p_{k i}$ is a point in $\sigma_{k i}$. The Riemann integral of a continuous $r$-form over a polyhedral $r$-chain $A=\sum a_{i} \sigma_{i}$, is defined by $\int_{A} \tau=\sum a_{i} \int_{\sigma_{i}} \tau$, where $\sum a_{i} \sigma_{i}$ is a simplicial subdivision of the polyhedral chain $A$.

An $r$-form in $E^{n}$ is bounded and measurable if all its components relative to a basis of $V$ are bounded and measurable. The Lebesgue integral of an $r$-form $\tau$ over an $r$-cell $\sigma$ is defined by

$$
\int_{\sigma} \tau=\int_{\sigma} \tau(p) \cdot \frac{\{\sigma\}}{|\sigma|} d p
$$

where $|\sigma|$ is the $r$-dimensional volume of $\sigma$ and the integral on the right is a Lebesgue integral of a real function. This is extended by linearity to domains that are polyhedral chains by

$$
\int_{A} \tau=\sum a_{i} \int_{\sigma_{i}} \tau
$$

if $A=\sum_{i} a_{i} \sigma_{i}$.

2.5. Stokes' theorem for polyhedral chains. The exterior derivative of a differentiable $r$-form $\tau$ is an $(r+1)$-form $d \tau$ defined by

$$
d \tau(p) \cdot\left(v_{1} \wedge \cdots \wedge v_{r+1}\right)=\sum_{i=1}^{r+1}(-1)^{i-1} \nabla_{v_{i}} \tau(p) \cdot\left(v_{1} \wedge \cdots \wedge \widehat{v}_{i} \wedge \cdots \wedge v_{r+1}\right)
$$

where $\widehat{v}_{i}$ denotes a vector that has been omitted, and $\nabla_{v_{i}}$ is a directional derivative operator. The last definition is represented using coordinates by

$$
(d \tau)_{\lambda_{1} \ldots \lambda_{r+1}}(p)=\sum_{i=1}^{r+1}(-1)^{i-1} \frac{\partial}{\partial x^{i}} \tau_{\lambda_{1} \ldots \hat{\lambda}_{i} \ldots \lambda_{r+1}}(p) .
$$

Stokes' theorem for polyhedral chains, based on the fundamental theorem of differential calculus, states that

$$
\int_{A} d \tau=\int_{\partial A} \tau
$$

for every differentiable $r$-form $\tau$ and an $(r+1)$-polyhedral chain $A$. 


\section{BANACH SPACES OF CHAINS}

3.1. Flat chains. The mass of a polyhedral $r$-chain $A=\sum a_{i} \sigma_{i}$ in $E^{n}$ is defined to be $|A|=\sum\left|a_{i}\right|\left|\sigma_{i}\right|$, where $\left|\sigma_{i}\right|$ denotes the the $r$-dimensional volume of $\left|\sigma_{i}\right|$. Thus, in case the interiors of the cells $\sigma_{i}$ of a polyhedral $r$-chain do not intersect, and if $a_{i}=1$, then the mass of the polyhedral chain is exactly its $r$-dimensional volume.

Definition 3.1. The flat norm, $|A|^{b}$, of a polyhedral $r$-chain $A$ in $E^{n}$ is defined by

$$
|A|^{\mathrm{b}}=\inf \{|A-\partial D|+|D|\}
$$

using all polyhedral $(r+1)$-chains $D$.

We note that it is not immediate that $|\cdot|^{b}$ is indeed a norm. Furthermore, the actual calculation of the the flat norm may be quite complicated even for simple $r$-chains. (For example, consider a 1-chain in the plane consisting two oriented line segments.) Taking $D=0$ above, it is clear that $|A|^{b} \leqslant|A|$.

Completing the space $\mathcal{A}_{r}\left(E^{n}\right)$ with respect to the flat norm gives a Banach space denoted by $\mathcal{A}_{r}^{b}\left(E^{n}\right)$. That is, $\mathcal{A}_{r}^{b}\left(E^{n}\right)$ contains the formal limits of all the sequences of polyhedral $r$-chains $A_{i}$, such that $\lim _{i \rightarrow \infty}\left|A_{i+1}-A_{i}\right|^{b}=0$. Elements of $\mathcal{A}_{r}^{b}\left(E^{n}\right)$ which are limits of such sequences are sometimes denoted by $\lim ^{b} A_{i}$. We refer to elements of $\mathcal{A}_{r}^{b}\left(E^{n}\right)$ as flat $r$-chains in $E^{n}$. If there are no intersections between cells and all coefficients have the value of 1 , we identify the flat chain with the set that contains its points.

Example 3.2. Consider the sequence of 1-chains $\left(A_{i}\right)$ in $E^{2}$ such that $A_{i}=L_{1 i}+L_{2 i}$ where $L_{1 i}$ and $L_{i 2}$ are 1-simplexes associated with two parallel line segments having the same length $L$, opposite orientation, and the line segment corresponding to $L_{2 i}$ is obtained from the line segment corresponding to $L_{1 i}$ by a translation of distance $d_{i}$ perpendicularly to its direction. If we take the rectangle generated by the two line segments as $D_{i}$ in the definition of the flat norm, it follows that $\left|A_{i}\right|^{b} \leqslant(L+2) d_{i}$. Thus, if $d_{i} \rightarrow 0$, the sequence $\left(A_{i}\right)$ converges to the zero chain in the flat norm. On the other hand, in the mass norm we have $\left|A_{i}-A_{i-1}\right|=2 L$ for all $i$ so the sequence does not converge. Roughly speaking, the geometrical significance of the flat norm is that, unlike the mass norm, it takes into account how closely the two segments are located.

If we let the length of the line segments shrink also so that for $A_{i}, L=d_{i}$, then by taking $D_{i}$ as above we get $\left|A_{i}\right|^{b} \leqslant d_{i}^{2}+2 d_{i}$ while taking $D_{i}=0$, implies $\left|A_{i}\right|^{b} \leqslant 2 d_{i}$ so $\left|A_{i}\right|^{b} \rightarrow 0$ as $d_{i}$.

Example 3.3. Consider the "staircase" sequence $\left(B_{i}\right)$ shown in Fig. 1. Here,

$$
A_{j}=\sum_{l=1}^{2^{j-1}} A_{j l}
$$

is the sum of $2^{j-1}$ oriented 1 -squares of size $d_{j}=1 / 2^{j}, B_{i}=B_{0}+\sum_{j=1}^{i} A_{j}$, and we take the limit as $i \rightarrow \infty$. Set for each square $A_{j l}$, the cell $D_{j l}$ such that $A_{j l}=\partial D_{j l}$. Then, using $D_{j l}$ in the definition of the flat norm we get $\left|A_{j l}\right|^{b} \leqslant d_{j}^{2}=2^{-2 j}$. Hence,

$$
\left|B_{i}-B_{i-1}\right|^{\mathrm{p}}=\left|A_{i}\right|^{\mathrm{b}} \leqslant 2^{i-1} 2^{-2 i}
$$

so the sequence $\left(B_{i}\right)$ converges.

Flat chains may be used to represent continuous and smooth submanifolds of $E^{n}$ and even irregular surfaces as shown above. As another example, starting with a triangle on $\mathbb{R}^{2}$ 


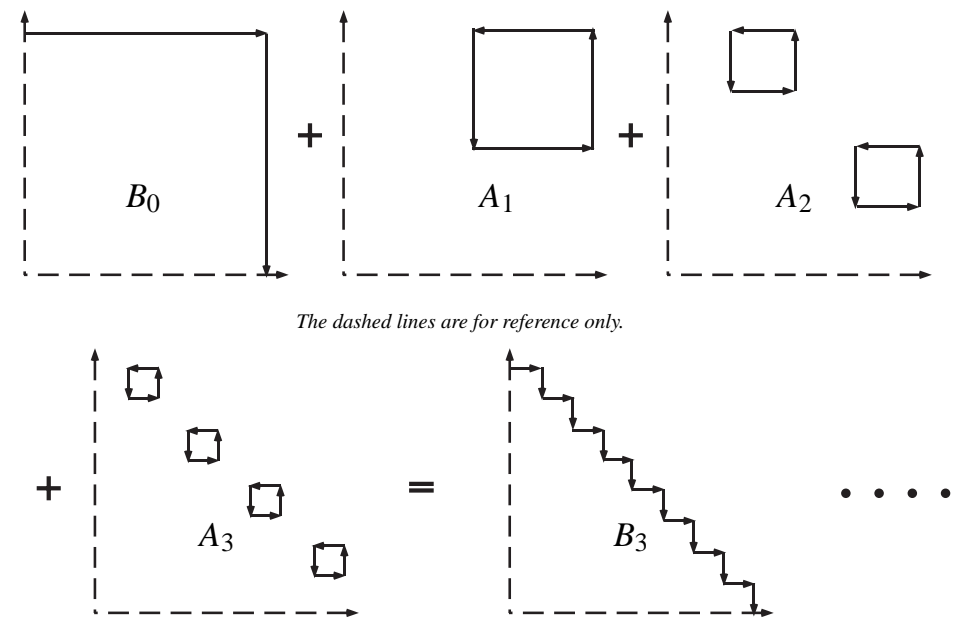

FIgURE 1. The staircase.

one may construct a plane in $\mathbb{R}^{3}$ by mapping the vertices using the values at the vertices of a real valued function $u$ on $\mathbb{R}^{2}$. One may subdivide the triangle and map the new vertices again using the mapping $u$ to construct a piecewise flat surface in $\mathbb{R}^{3}$ approximating the graph of $u$. This procedure may be repeated to construct a sequence of 2-chains. If for the function $u$ one uses a continuous function that is nowhere differentiable one obtains a flat chain that represents a surface that is not rectifiable.

The Riemann integral of a continuous $r$-form $\tau$ over a flat $r$-chain $A=\lim A_{i}$, is defined to be $\int_{A} \tau=\lim \int_{A_{i}} \tau$, if the limit exists.

The boundary of a flat $(r+1)$-chain $A=\lim ^{b} A_{i}$, is defined to be $\partial A=\lim \partial A_{i}$. The boundary of a flat $(r+1)$-chain always exists as a flat $r$-chain.

3.2. Sharp chains. Whitney obtained chains that are even less regular then the flat chains by introducing a possibly smaller norm. Thus, more Cauchy sequences will converge and one ends up with a larger completed space.

Definition 3.4. The sharp norm $|A|^{\sharp}$ of a polyhedral $r$-chain $A=\sum a_{i} \sigma_{i}$ is defined by

$$
|A|^{\sharp}=\inf \left\{\frac{\sum\left|a_{i}\right|\left|\sigma_{i}\right|\left|v_{i}\right|}{r+1}+\left|\sum a_{i} \operatorname{trans}_{v_{i}} \sigma_{i}\right|^{b}\right\}
$$

using all vectors $v_{i} \in E^{n}$, where trans $v$ is a translation operator that moves each point $p$ of $\sigma$ to $p+v$, giving a translated cell trans $v \sigma$ with the same orientation as $\sigma$.

Clearly, setting all $v_{i}=0$, we conclude that $|A|^{\sharp} \leqslant|A|^{b}$ so the sharp norm defines a coarser topology. Completing the space $\mathcal{A}_{r}\left(E^{n}\right)$ with respect to the sharp norm gives a Banach space denoted by $\mathcal{A}_{r}^{\sharp}\left(E^{n}\right)$ whose elements are referred to as sharp chains. It follows that $\mathcal{A}_{r}^{\mathrm{b}}\left(E^{n}\right)$ is a Banach subspace of $\mathcal{A}_{r}^{\sharp}\left(E^{n}\right)$.

Example 3.5. Consider again the sequence of pairs of 1-vectors in $\mathbb{R}^{2}$ of length $d_{i}$ situated a distance $d_{i}$ apart as above. Taking $v_{1}=0$, and $v_{2}$ as the vector such that trans $v_{v_{2}}$ will cause the two line segments to overlap so $\left|v_{2}\right|=d_{i}$, we have $\left|A_{i}\right|^{\sharp} \leqslant d_{i}^{2} / 2$. Hence, for $d_{i} \rightarrow 0$, the sharp norm of the shrinking pairs tends to zero faster than the flat norm. 
Consider the "staircase strainer" sequence $\left(B_{i}\right)$ constructed in the unit square as shown in Fig. 2. Here, $A_{j}$ is the sum of $2^{j-1}$ pairs of size $d_{j}=1 / 2^{j}, B_{i}=B_{0}+\sum_{j=1}^{i} A_{j}$, and we take the limit as $i \rightarrow \infty$. For the flat norm we have

$$
\left|B_{i}-B_{i-1}\right|^{b}=\left|A_{i}\right|^{b} \leqslant 2^{i-1} 2 / 2^{i}=1,
$$

so the sequence $\left(B_{i}\right)$ does not converge. On the other hand, for the sharp norm

$$
\left|B_{i}-B_{i-1}\right|^{\sharp}=\left|A_{i}\right|^{\sharp} \leqslant 2^{i-1}\left(1 / 2^{i}\right)^{2} / 2=2^{-i} / 4,
$$

and the sequence converges. Thus, we will be able to calculate the total flux through the staircase strainer limit. (The extensive property under consideration may flow through the strainer $B_{i}$ at the horizontal segments only.)

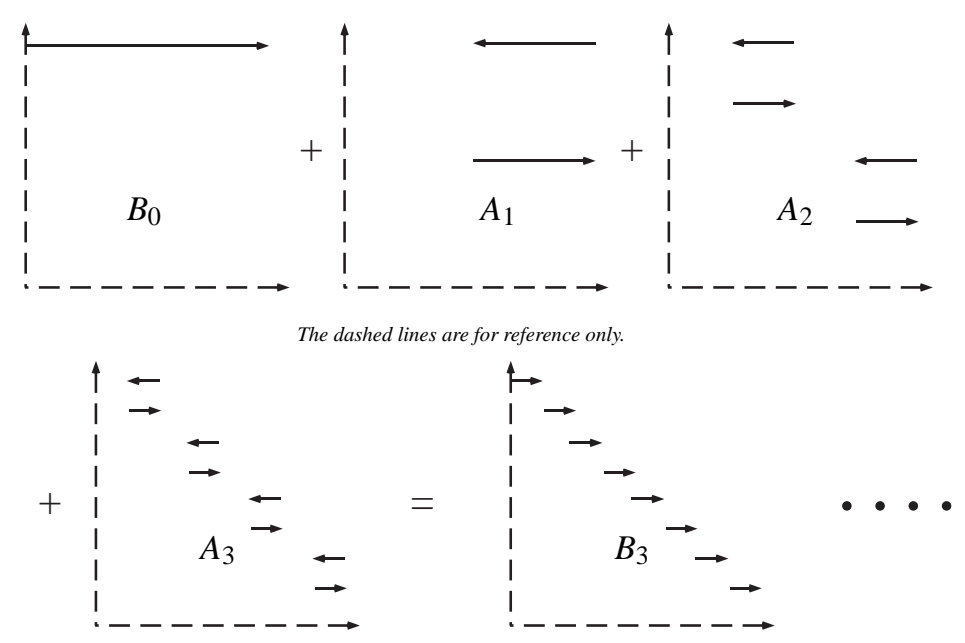

FIGURE 2. The staircase strainer

Similarly, the "staircase mixer" sequence shown in Fig. 3 converges in the sharp norm but not in the flat norm.

Roughly speaking, the difference in behavior between the flat norm and the sharp norm may be described as follows. Consider a sequence $\left(A_{i}\right)$ of shrinking $r$-polyhedral chains of typical size $s_{i} \rightarrow 0$. If $A_{i}$ is the boundary $\partial B_{i}$ of a shrinking $(r+1)$-chain $B_{i}$, then taking $D=B_{i}$ in the definition of the flat norm, $\left|A_{i}\right|^{b}$ shrinks like $s_{i}^{r+1}$. If $A_{i}$ cannot be represented as the boundary of an $(r+1)$-chain, the $r$-dimensional mass of some subset of $A_{i}$ will always be present in the definition of the flat norm and hence $\left|A_{i}\right|^{b}$ will shrink like $s_{i}^{r}$ only. On the other hand, for the sharp norm, if one can cancel the flat norm of a chain by translating simplexes by vectors of the same order of magnitude as $s_{i}$, then the price to pay in the definition of the sharp norm is bounded by $s_{i}^{r+1}$ whether $A_{i}$ is the boundary of another chain or not.

The Riemann integral of a continuous $r$-form $\tau$ over a sharp $r$-chain $A=\lim A_{i}$, is defined to be $\int_{A} \tau=\lim \int_{A_{i}} \tau$, if the limit exists.

It is noted that being less regular than a flat chain, the boundary of a sharp chain need not exist as a sharp chain. 


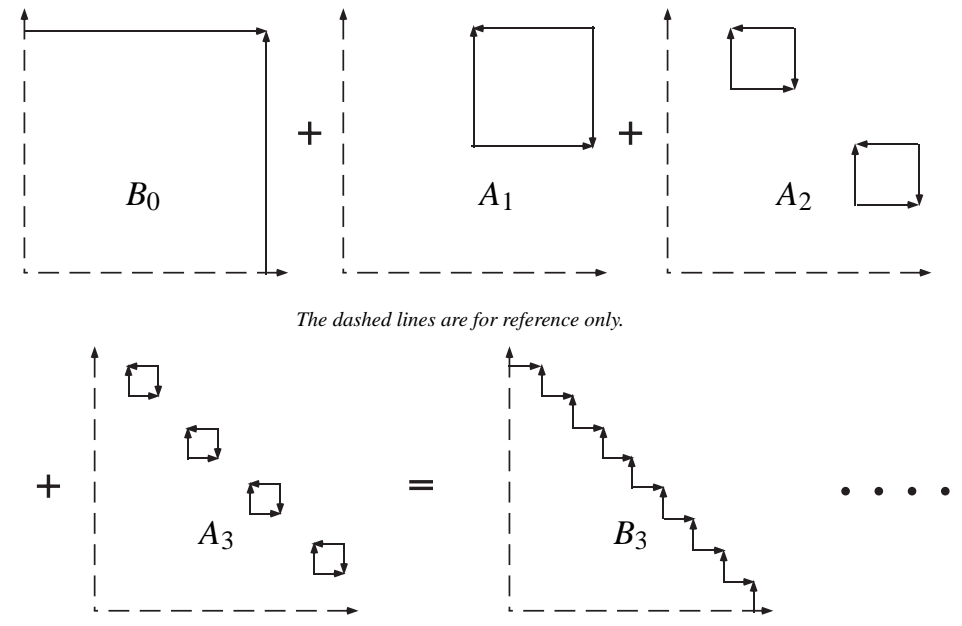

FIGURE 3. The staircase mixer

3.3. Natural chains. A basic notion of Harrison's constructions is that of a dipole. A simple $r$-dimensional 0 -dipole is an $r$-simplex $\sigma^{0}$ whose diameter $\operatorname{diam}\left(\sigma^{0}\right) \leqslant 1$. A simple $r$-dimensional 1-dipole is a chain of the form $\sigma^{1}=\sigma^{0}-\operatorname{trans}_{v_{1}} \sigma^{0}$ for a vector $v_{1}$, such that $\left|v_{1}\right| \leqslant 1$, and $\operatorname{trans}_{v_{1}} \sigma^{0}$ is disjoint from $\sigma^{0}$. Inductively, a simple $r$-dimensional $j$-dipole is an $r$-chain of the form $\sigma^{j}=\sigma^{j-1}-\operatorname{trans}_{v_{j}} \sigma^{j-1}$, where $\sigma^{j-1}$ is a simple $r$-dimensional $(j-1)$-dipole, and $v_{j}$ is a vector with $\left|v_{j}\right| \leqslant 1$ such that $\operatorname{trans}_{v_{j}} \sigma^{j-1}$ is disjoint from $\sigma^{j-1}$. A simple $j$-dipole is therefore determined by the simplex $\sigma^{0}$ and the $v_{1}, \ldots, v_{j}$ vectors. A $j$-dipole is a simplicial chain

$$
D^{j}=\sum_{i} a_{i} \sigma_{i}^{j}
$$

of simple $j$-dipoles.

Given a simple $j$-dipole $\sigma^{j}$ constructed by the simplex $\sigma^{0}$ and vectors $v_{1}, \ldots, v_{j}$, its $j$-dipole mass is defined by

$$
\left|\sigma^{j}\right|_{j}=\left|\sigma^{0}\right|\left|v_{1}\right| \cdots\left|v_{j}\right|
$$

$\left(\left|\sigma^{0}\right|\right.$ is the mass of $\left.\sigma^{0}\right)$. The $j$-dipole mass of the $j$-dipole $D^{j}=\sum_{i} a_{i} \sigma_{i}^{j}$ is defined as

$$
\left|D^{j}\right|_{j}=\sum_{i}\left|a_{i}\right|\left|\sigma_{i}^{j}\right|_{j} .
$$

Using the notion of a dipole and the dipole mass, the $k$-natural norm, $k=1,2, \ldots$, on the space of polyhedral chains is defined by

$$
|A|_{k}^{\natural}=\inf \left\{\sum_{s=0}^{k}\left|D^{s}\right|_{s}+|C|_{k-1}^{\natural}\right\},
$$

where the infimum it taken over all decompositions of $A$ in the form $A=\sum_{s=0}^{k} D^{s}+\partial C$, for $s$-dipoles $D^{s}$. The Banach space one obtains by completing the space of polyhedral chains relative to this norm is denoted by $\mathcal{A}_{r}^{k}$ and its elements are referred to as k-natural $r$-chains. Clearly, the 0-natural norm is equivalent to the flat norm. Harrison also defines 
norms associated with fractional values of $r$ that are related to the Hölder conditions but we omit the discussion of such chains here.

As $k$ increases, the the spaces of natural chains become larger, i.e., $\mathcal{A}_{r}^{k}$ is a Banach subspace of $\mathcal{A}_{r}^{l}$ for $k<l$. For increasing values of $k$ these spaces contain increasingly irregular chains. For example, various fractals are natural chains, and the $k$-th distributional derivative of the Dirac measure on the real line belongs to $\mathcal{A}_{1}^{k+1}$ (see [8]).

For a $k$-natural $r$-chain $A$, let $\tau$ be a form on $A$ that has $k-1$ bounded derivatives and whose $k$-th derivative is Lipschitz. The Riemann integral of $\tau$ over a natural $r$-chain $A=\lim ^{\natural} A_{i}$, is defined to be $\int_{A} \tau=\lim \int_{A_{i}} \tau$. Indeed, Harrison shows that the limit always exists as integrals over polyhedral chains are bounded by the natural norms of the chains.

A clear advantage of using the natural norms in comparison with the sharp norm is the behavior of the natural chains under the boundary operator: the boundary operator of polyhedral chains extends to a continuous linear operator $\partial: \mathcal{A}_{r}^{k} \rightarrow \mathcal{A}_{r-1}^{k-1}$.

\section{Cochains}

Cochains are elements of the dual spaces to the Banach spaces of flat, sharp, and natural chains. The basic idea of the application of Whitney's abstract integration theory to the analysis of Cauchy fluxes is that cochains in the various dual spaces are abstract counterparts of total fluxes. Specifically, for classical continuum mechanics we regard the total flux $T_{A}$ of a certain extensive property $P$ through a 2-dimensional domain $A$ in $E^{3}$ as the action $T \cdot A$ of a 2 -cochain $T$ on the 2 -chain $A$ associated with the domain. For the sake of simplicity of the notation we used here the same notation for both the domain and the representing chain. It is noted that chains contain more information than just the domain where they are supported. For example, any continuous function defined on a submanifold of the Euclidean space may be represented as a chain. Obviously, the coefficients for the simplexes that make up the chain will be different than 1 and will represent the values of the function. In such a case, if we interpret the value of the function as a component of a velocity field, the action of a cochain on the chain may be interpreted as the calculation of power. Thus, geometric integration theory combines the classical approaches to flux theory and the variational weak approach. An immediate benefit of using geometric integration theory is that the analysis holds for $r$-chains in $E^{n}$ for all values of $r \leqslant n$.

The properties of cochains that make them suitable mathematical models for Cauchy fluxes follow firstly from the linearity of their action on chains which is common to all Banach spaces considered above. Linearity of the action of cochains implies both the additivity and the action-interaction-antisymmetry properties assumed in various formulations of continuum mechanics. For example, given a cochain $T$, we have $T \cdot(-A)=-T \cdot A$. Secondly, the properties of the various cochains are determined by the continuity of their action on chains which is directly linked to the norm on the respective space of chains. Basic observations regarding the relations between the various norms and the properties we expect fluxes to have will described below.

4.1. Flat cochains. Flat $r$-cochains in $E^{n}$ are the elements of $\mathcal{A}_{r}^{b}\left(E^{n}\right)^{*}$, the dual space of $\mathcal{A}_{r}^{b}\left(E^{n}\right)$. We will see next how the topology induced by the flat norm is related to traditional assumptions of Cauchy flux theory. We recall that in various formulations of Cauchy's flux theory it is assumed that the total flux is bounded by both the volume and area of the corresponding region. That is, there are positive numbers $N_{1}$ and $N_{2}$ such that 
for every region $A$,

$$
\left|T_{\partial A}\right| \leqslant N_{2}|\partial A|, \quad\left|T_{\partial A}\right| \leqslant N_{1}|A|,
$$

where we use the mass norm to denote both the area and volume of the respective sets. In terms of a cochain $T$ these boundedness conditions will be written as

$$
|T \cdot A| \leqslant N_{2}|A|, \quad|T \cdot \partial D| \leqslant N_{1}|D|,
$$

for any $r$-chain $A$ and an $(r+1)$-chain $D$. Thus,

$$
\begin{aligned}
|T \cdot A| & =|T \cdot A-T \cdot \partial D+T \cdot \partial D| \\
& \leqslant|T \cdot A-T \cdot \partial D|+|T \cdot \partial D| \\
& \leqslant N_{1}|A-\partial D|+N_{2}|D| \\
& \leqslant C_{T}(|A-\partial D|+|D|),
\end{aligned}
$$

where $C_{T}$ is the least upper bound of all positive numbers satisfying this relation for all $(r+1)$-chains $D$. The basic idea is to look at this relation as a requirement of continuity, $|T \cdot A| \leqslant C_{T}\|A\|$, for the linear operator $T$. Since $D$ is arbitrary it is natural to set then

$$
|A|^{b}=\inf _{D}\{|A-\partial D|+|D|\} .
$$

It follows that the flat norm is the smallest of all norms that make the flux operators satisfying the boundedness condition continuous. As such, upon the completion of the space of polyhedral chains with respect to the flat norm, we obtain the largest Banach space for which the bounded flux operators are continuous. This means that flat chains are the most general geometrical objects for which the action of bounded flux operators is continuous.

Conversely, if we consider norms $|\cdot|^{x}$ on the space of polyhedral chains and wish to consider the action of a continuous flux functional $T$, then, $|T \cdot A| \leqslant C_{T}|A|^{x}$. If one requires that $|A|^{x} \leqslant|A|$ and $|\partial D|^{x} \leqslant|D|$, for any $r$-chain $A$ and $(r+1)$-chain $D$, then the boundedness conditions are implied by continuity because

$$
|T \cdot A| \leqslant C_{T}|A|^{x} \leqslant C_{T}|A|, \quad \text { and } \quad|T \cdot \partial D| \leqslant C_{T}|\partial D|^{x} \leqslant C_{T}|D| .
$$

In order to admit the most general flux operators that satisfy these conditions we need the largest norm such that $|A|^{x} \leqslant|A|$ and $|\partial D|^{x} \leqslant|D|$. Indeed it can be shown that the flat norm is the largest norm satisfying these two conditions.

4.2. Sharp cochains. Sharp $r$-cochains in $E^{n}$ are elements of $\mathcal{A}_{r}^{\sharp}\left(E^{n}\right)^{*}$, the dual the space of $\mathcal{A}_{r}^{\sharp}\left(E^{n}\right)$. Since flat chains form a Banach subspace of $\mathcal{A}_{r}^{\sharp}\left(E^{n}\right)$, every sharp cochain may be restricted to flat chains. In other words, any sharp cochain is also flat.

The additional property of sharp cochains that distinguishes them from flat cochains is the boundedness under translation. Given a sharp cochain $T$, consider for an $r$-cell $\sigma$ and a vector $v$, the difference in the flux due to the translation by $v$, i.e., $\left|T \cdot \sigma-T \cdot \operatorname{trans}_{v} \sigma\right|$. The continuity of $T$ implies that

$$
\begin{aligned}
\left|T \cdot \sigma-T \cdot \operatorname{trans}_{v} \sigma\right| & \leqslant C_{T}\left|\sigma-\operatorname{trans}_{v} \sigma\right|^{\sharp} \\
& \leqslant C_{T} \frac{|\sigma||v|}{r+1},
\end{aligned}
$$

by choosing $v_{1}=0$ and $v_{2}=-v$ in the definition of the sharp norm. Thus, continuity implies that there is a positive $N_{3}$ such that

$$
\left|T \cdot \sigma-T \cdot \operatorname{trans}_{v} \sigma\right| \leqslant N_{3}|\sigma||v| .
$$

In particular, the difference tends to zero if so does the magnitude of $v$. Clearly, this imposes a regularity restriction on sharp cochains. In analogy with flat chains, the sharp norm 
is the smallest of all norms for which all the flux operators satisfying the earlier boundedness conditions and boundedness under translation are continuous. Hence, in comparison with all other norms, it allows more elements to be added to the space of polyhedral chains in the process of completion.

Conversely, if we consider norms $|\cdot|^{x}$ on the space of polyhedral chains and wish to consider the action of a continuous flux functional $T$, then, $|T \cdot A| \leqslant C_{T}|A|^{x}$. If one requires that $|A|^{x} \leqslant|A|,|\partial D|^{x} \leqslant|D|$ and $\mid \sigma-$ trans $\left._{v} \sigma\right|^{x} \leqslant|\sigma||v|$, for every $r$-chain $A$, $(r+1)$-chain $D, r$-cell $\sigma$, and vector $v$, then the boundedness conditions are implied by continuity. For example, boundedness under translation is implied by

$$
\left|T \cdot \sigma-T \cdot \operatorname{trans}_{v} \sigma\right| \leqslant C_{T}\left|\sigma-\operatorname{trans}_{v} \sigma\right|^{x} \leqslant C_{T}|\sigma||v| .
$$

In order to admit the most general flux operators that satisfy these three conditions we need the largest norm satisfying them. Indeed it can be shown that the sharp norm is the largest norm satisfying the conditions.

4.3. Natural cochains. A $k$-natural $r$-cochain is an element of $\mathcal{A}_{r}^{k *}$, the dual space of $\mathcal{A}_{r}^{k}$. Since the natural norms $|\cdot|_{k}^{\natural}$ are smaller than the flat norm for $k>0$, all natural cochains are flat cochains. In fact, we will see later that natural cochains are very regular. It is a basic guiding principle in geometric integration that as chains become increasingly irregular the cochains become increasingly regular.

\section{REPRESENTATION OF COCHAINS,} THE ISOMORPHISM THEOREM AND FLUXES

5.1. The Cauchy mapping. The Cauchy mapping of $r$-directions induced by an $r$-cochain is completely analogous to the mapping that gives the dependence of the flux density on the unit normal in classical continuum mechanics, hence the terminology we use. Let the $r$-direction $\alpha$ of an $r$-cell $\sigma$ be the $r$-vector $\{\sigma\} /|\sigma|$. The Cauchy mapping $D_{T}$, associated with the cochain $T$ is defined to be the function of points and $r$-directions such that

$$
D_{T}(p, \alpha)=\lim _{i \rightarrow \infty} T \cdot \frac{\sigma_{i}}{\left|\sigma_{i}\right|},
$$

where $\sigma_{i}$ is a sequence of $r$-cells containing $p$ with $r$-direction $\alpha$ such that

$$
\lim _{i \rightarrow \infty} \operatorname{diam}\left(\sigma_{\mathrm{i}}\right)=0 .
$$

As the $r$-direction $\alpha$ is the analog of the unit normal $\boldsymbol{n}$ used in continuum mechanics, the analog of Cauchy's flux theorem will be the assertion that the restriction of the Cauchy mapping to each point $p$ may be extended to a linear mapping of $r$-vectors. In other words, $D_{T}$ is a form in $E^{n}$.

5.2. The representation theorem for sharp fluxes. The analog to Cauchy's flux theorem in Whitney's geometric integration theory for sharp cochains states the following.

Proposition 5.1. For each sharp $r$-cochain $T$, the Cauchy mapping $D_{T}$ may be extended to a unique $r$-form that represents $T$ by

$$
T \cdot A=\int_{A} D_{T}
$$

for every polyhedral chain $A$. 
Clearly, the proposition defines the integral of a form over a sharp chain by continuity.

Whitney's theory determines exactly the forms that represent sharp cochains- the sharp forms. Firstly, the norm $|\cdot|_{0}$ is defined on $V^{r}$ by

$$
|\tau|_{0}=\sup \{|w \cdot \alpha| \mid \alpha \text { simple, }|\alpha|=1\} .
$$

The sharp norm of the form $\tau$ is defined by

$$
|\tau|^{\sharp}=\sup _{p, q \in E^{n}}\left\{|\tau(p)|_{0},(r+1) \frac{|\tau(q)-\tau(p)|_{0}}{|q-p|}\right\}
$$

Then, a sharp form is defined to be a form whose sharp norm is finite. Thus, sharp forms are bounded Lipschitz forms. Using the norm topology on the space of cochains where

$$
|T|^{\sharp}=\sup _{|A|^{\sharp}=1}|T \cdot A|,
$$

it can be shown that the previous proposition defines an isomorphism of the Banach space of sharp cochains and the Banach space of sharp forms.

5.3. Representation of flat cochains. While sharp $r$-cochains are regular enough to be represented uniquely by sharp $r$-forms, flat $r$-cochains are less regular, and each flat $r$ cochain is represented by an equivalence class of $r$-forms which satisfy certain regularity conditions. Sharp forms representing sharp cochains are continuous and Riemann integration may be used. The representation of flat cochains by forms uses the analogous Lebesgue integration.

The Lebesgue integral of an $r$-form over a flat $r$-chain $A=\lim ^{b} A_{i}$ is defined by

$$
\int_{A} \tau=\lim \int_{A_{i}} \tau
$$

if the limit exists. (The integrals on the right-hand side are Lebesgue integrals on polyhedral chains defined earlier.)

The analysis of the representation of flat cochains by forms requires more attention then the sharp counterpart. For example, in the definition of the Cauchy mapping

$$
D_{T}(p, \alpha)=\lim _{i \rightarrow \infty} T \cdot \frac{\sigma_{i}}{\left|\sigma_{i}\right|},
$$

it is required that in the converging sequence $\left(\sigma_{i}\right)$, each of the simplexes will contain $p$ as a vertex. It turns out that for each $r$-direction $\alpha, D_{T}(p, \alpha)$ is defined almost everywhere. Wolfe's representation theorem as formulated by Whitney [23, p. 261] for flat cochains states as follows.

Proposition 5.2. Let $T$ be a flat $r$-cochain in an open set $R \subset E^{n}$. Then, there is a set $Q \subset R$, with $|R-Q|=0$, such that for each $p \in Q, D_{T}(p, \alpha)$ is defined for all $r$ directions $\alpha$, and is extendable to all $r$-vectors, giving an $r$-covector $D_{T}(p)$. The $r$-form $D_{T}$ is bounded and measurable in $R$. For any $r$-simplex $\sigma$ in $R, D_{T}$ is a measurable $r$-form relative to the plane of $\sigma$ and

$$
T \cdot \sigma=\int_{\sigma} D_{T}
$$

In fact, one can describe exactly the flat forms - those forms that represent flat cochains. The exact conditions such that any flat $r$-form is associated with a unique flat $r$-cochain use the notions of $Q$-good simplexes and association of a form with a flat cochain. In order to avoid the technical details and since we are mainly interested in the existence of the representing forms, these notions will not be presented here (see [23, pp. 263-266] for 
the details). As one would expect, a flat cochain is associated with an equivalence class of forms under equality almost everywhere. The quotient space of flat forms obtained by identifying forms that are equal almost everywhere together with an appropriate norm, the flat norm of forms, is isomorphic to the space of flat cochains.

5.4. Representation of natural cochains. As mentioned earlier, natural cochains are regular. Harrison's representation theorem states that for $k>0$ every $k$-natural cochain $T$ is represented by a unique differential form $D_{T}$ as

$$
T \cdot A=\int_{A} D_{T} .
$$

where the first $k$ derivatives of $D_{T}$ are bounded and the $k$-th derivative is Lipschitz. In fact, this relation defines an isomorphism of the of the space of $k$-natural cochains and the space of differential forms having this degree of smoothness (equipped with the suitable $C^{k \text { Lip }}$-norm).

\section{CoBOUNDARIES AND DIFFERENTIAL BALANCE EQUATIONS}

Coboundaries generalize exterior differentiation and their definition is purely algebraic. The coboundary $d T$ of an $r$-cochain $T$ is the $(r+1)$-cochain defined by

$$
d T \cdot A=T \cdot \partial A,
$$

i.e., it is the dual of the boundary operator for chains. As $\partial(\partial A)=0$, one has $d(d T)=0$. The basic result concerning coboundaries is that the coboundary of a flat cochain is flat and the same holds for the coboundary of a sharp cochain. This implies a very general formulation of the balance equation. For a cochain $T$ that is either sharp or flat, the coboundary exists as a flat cochain and we may define an $(r+1)$-cochain $S$, satisfying $d T+S=0$, so the balance equation $S \cdot A+T \cdot \partial A=0$ holds. Here, $S$ is interpreted as the cochain giving the rate of change of total amount of the property $P$ in the flat $(r+1)$-chain $A$ (assuming there is no source term).

If the form $D_{T}$ representing the cochain $T$ is differentiable, then the flat form $D_{d T}$ representing $d T$ is given as the exterior derivative of $D_{T}$ as one would expect, i.e.,

$$
D_{d T}=d D_{T} .
$$

Thus, using $\tau$ for $D_{T}$, the abstract balance equation above assumes the form

$$
d \tau+b=0, \quad \int_{A} b+\int_{\partial A} \tau=0 .
$$

In the more general case where $\tau$ is an arbitrary flat form representing the flat cochain $T$, $d T$ is a flat cochain and hence it may be represented by any flat form $d_{0} \tau$ in the equivalence class of $D_{d T}$. Thus, one may write the "differential" balance in the general situation of flat cochains. In fact,

$$
|T|^{\mathrm{b}}=\sup _{p}\left\{\left|D_{T}(p)\right|,\left|D_{d T}(p)\right|\right\} .
$$

The right-hand side of this identity is the flat norm of the form $D_{T}$.

In the particular case where $T$ is a sharp cochain represented by the sharp form $\tau=D_{T}$, the functions giving the components of $\tau$ are Lipschitz mappings, hence, it has an analytic exterior derivative $d \tau$ as in Subsection 2.5 almost everywhere. Furthermore, it turns out that $d_{0} \tau=d \tau$ almost everywhere. 
6.1. Coboundaries for natural cochains. The fact that the boundary operator is continuous for natural chains allows the definition of the coboundary operator as the dual of the boundary operator. For natural cochains, one has $D_{d T}=d D_{T}$. It is noted that for natural cochains one may use a geometric definition of the exterior derivative as follows. Let $p$ be a point and $\alpha$ an $r$-direction, then taking a decreasing sequence of $r$-simplexes $\left(\sigma_{i}\right)$ containing $p$, all of which are in the direction of $\alpha$, then

$$
d \tau(p, \alpha)=\lim _{\left|\sigma_{i}\right| \rightarrow 0} \frac{\int_{\partial \sigma_{i}} \tau}{\left|\sigma_{i}\right|} .
$$

Thus, the balance equation holds pointwise.

Acknowledgements. The research leading to this paper was partially supported by a Kreitman Doctoral Fellowship to G. Rodnay and by the Paul Ivanier Center for Robotics Research and Production Management at Ben-Gurion University.

\section{REFERENCES}

[1] M. Degiovanni, A. Marzocchi, and A. Musesti. Cauchy fluxes associated with tensor fields having divergence measure. Archive for Rational Mechanics and Analysis, 147:197-223, 1999.

[2] H. Federer. Geometric Measure Theory. Springer Verlag, New York, 1969.

[3] R. L. Fosdick and E. G. Virga. A variational proof of the stress theorem of Cauchy. Archive for Rational Mechanics and Analysis, 105:95-103, 1989.

[4] M. E. Gurtin and L. C. Martins. Cauchy's theorem in classical physics. Archive for Rational Mechanics and Analysis, 60:305-324, 1975.

[5] M. E. Gurtin and W. O. Williams. An axiomatic foundation for continuum thermodynamics. Archive for Rational Mechanics and Analysis, 26:83-117, 1967.

[6] M. E. Gurtin, W. O. Williams, and W. P. Ziemer. Geometric measure theory and the axioms of continuum thermodynamics. Archive for Rational Mechanics and Analysis, 92:1-22, 1986.

[7] J. Harrison. Stokes' theorem for nonsmooth chains. Bulletin of the American Mathematical Society, 29(2):235-242, 1993.

[8] J. Harrison. Continuity of the integral as a function of the domain. Journal of Geometric Analysis, 8(5):769$795,1998$.

[9] J. Harrison. Isomorphisms of differential forms and cochains. Journal of Geometric Analysis, 8(5):797-807, 1998.

[10] J. Harrison. Flux across nonsmooth boundaries and fractal Gauss/Green/Stokes theorems. Journal of Physics A, 32(28):5317-5327, 1999.

[11] W. Noll. The Foundations of Classical Mechanics in Light of Recent Advances in Continuum Mechanics, in The Axiomatic Method, with Special Reference to Geometry and Physics, pages 265-281. North-Holland, Amsterdam (Symposium at Berkeley, 1957), 1959.

[12] W. Noll. Lectures on the foundations of continuum mechanics and thermodynamics. Archive for Rational Mechanics and Analysis, 52:61-92, 1973.

[13] W. Noll. Continuum mechanics and geometric integration theory. In Categories in Continuum Physics, volume 1174 of Lecture Notes in Mathematics, pages 17-29. Springer Verlag, 1986.

[14] W. Noll and E. G. Virga. Fit regions and functions of bounded variation. Archive for Rational Mechanics and Analysis, 102:1-21, 1988.

[15] R. Segev. Forces and the existence of stresses in invariant continuum mechanics. Journal of Mathematical Physics, 27(1):163-170, 1986.

[16] R. Segev. The geometry of Cauchy‘s fluxes. Archive for Rational Mechanics and Analysis, 154:183-198, 2000.

[17] R. Segev. A correction of an inconsistency in my paper 'Cauchy's theorem on manifolds'. Journal of Elasticity, 63:55-59, 2002.

[18] R. Segev and G. deBotton. On the consistency conditions for force systems. International Journal of Nonlinear Mechanics, 26(1):47-59, 1991

[19] R. Segev and G. Rodnay. Cauchy's theorem on manifolds. Journal of Elasticity, 56:129-144, 1999.

[20] M. Šilhavý. The existence of the flux vector and the divergence theorem for general Cauchy fluxes. Archive for Rational Mechanics and Analysis, 90:195-212, 1985. 
[21] M. Šilhavý. Cauchy's stress theorem and tensor fields with divergences in $L^{p}$. Archive for Rational Mechanics and Analysis, 116:223-255, 1991.

[22] H. Whitney. Algebraic topology and integration theory. Proceedings of the National Academy of Sciences, 33:1-6, 1947.

[23] H. Whitney. Geometric Integration Theory. Princeton University Press, Princeton, New Jersey, 1957.

[24] J.H. Wolfe. Tensor Fields Associated with Lipschitz Cochains. PhD thesis, Harvard, 1948.

Department of Mechanical Engineering,, Ben-Gurion University, P.O.Box 653,, BeerSHEVA 84105, ISRAEL,, E-MAIL: RSEGEV@BGUMAIL.BGU.AC.IL 